\title{
Role of Multi-Slice Computed Tomography and Magnetic Resonance Imaging in Evaluation of Laryngeal Tumors
}

\author{
ISRAA S. A. HEDIA, M.Sc.*; REDA A. EL-ARABAWY, M.D.*; MOHAMED N. EL-SHEIKH, M.D.** and \\ ATEF H. TEAMA, M.D.* \\ The Departments of Radiology \& Medical Imaging* and Otorhinolaryngology (ORL)**, Faculty of Medicine, Tanta University, \\ Tanta, Egypt
}

\begin{abstract}
Background: Imaging of laryngeal tumors is crucial for evaluation, preparing, treatment and for ensuring proper follow-up. Computed tomography and magnetic resonance imaging are the main imaging tools. Each modality showed its own advantages for assessment of local, regional or distant staging.
\end{abstract}

Aim of Study: The aim of this study was to evaluate the role of multi slice computed tomography and magnetic resonance imaging in diagnosis of laryngeal tumors.

Material and Methods: This prospective study included (30) patients in different age groups who were highly suspected with laryngeal tumors or known to be having laryngeal tumor. Patients were referred by Oto Rhino Laryngology (ORL) Department of Tanta University Hospital to the CT Unit \& MRI Units of the Diagnostic Radiology \& Medical Imaging Department, Tanta University Hospitals. All cases were subjected to full history, complete physical examination, radiological \& medical imaging examination at which all cases were exposed to CT scanning of the neck while only 22 cases of them performed additional MRI examination of the neck. Further endoscopic examination and histopathological correlation were done. Data was analyzed by using SPSS.

Results: MRI showed higher accuracy than $\mathrm{CT}$ as regards to pathological T staging of laryngeal tumors 244 that showed $86.4 \%$ for MRI while $66.7 \%$ for CT. Both CT and MRI were equal in evaluation of certain areas as anterior commissure and pyriform sinus while MRI showed higher accuracy in evaluation of other laryngeal subsites namely preepiglottic, paraglottic spaces, posterior commissure, epiglottis, vocal cords, thyroid cartilage extralaryngeal spread.and metastatic lymphadenopathy with the total accuracy higher in MRI than in CT.

Conclusion: MRI could be considered a helpful diagnostic method for pre and post-therapeutic staging of laryngeal tumors specially early glottic lesions and the combined utility of CT and MRI could help to overcome the disadvantages and improve the accuracy specially about thyroid cartilage assessment and $\mathrm{T}$ staging of laryngeal carcinomas.

Correspondence to: Dr. Israa S.A. Hedia, The Department of Radiology \& Medical Imaging, Faculty of Medicine, Tanta University, Tanta, Egypt
Key Words: Laryngeal tumors - SCC - Glottic - MSCT MRI.

\section{Introduction}

TUMORS of the larynx either benign or malignant constitute about $25 \%$ of all head and neck tumors and about $1-5 \%$ of all malignancies diagnosed annually. Laryngeal cancer is the second most common respiratory cancer after lung cancer. Its incidence is increasing over time in much of the world and this increase is generally accepted to be related to changes in tobacco and alcohol consumption $[1,2]$.

Integration of endoscopic findings with crosssectional imaging to assess the submucosal, paralaryngeal spaces, extralaryngeal spread and cartilaginous invasion of these tumors improves the $\mathrm{T}$ staging accuracy and influences the treatment decisions in these patients. Imaging also provides information about the nodal disease, systemic metastases, any synchronous tumors and recurrent disease [3-5].

Computed Tomography (CT) used to be the examination of choice that is performed for the evaluation of pathological laryngeal lesions due to its wide accessibility, shorter scanning time as nearly free of movement artifacts, aimed especially for patients who require immediate diagnosis (large obstructive lesions) or for uncooperative patients [6].

Magnetic Resonance (MR) imaging with use of various sequences has been shown to be more accurate than $\mathrm{CT}$ in specific situations such as assessing soft tissue, cartilaginous invasion, preepiglottic space and tongue base invasion [7]

MR imaging creates geometric distortion at the edges of the field of view and susceptibility artifacts 
at interfaces between bone and air. MRI is more sensitive than CT in the diagnosis of cartilaginous invasion [8], however, most institutions continue to use $\mathrm{CT}$ as the primary cross-sectional modality as advances in high-resolution multi-slice $\mathrm{CT}$ have kept CT competitive with MRI [9].

\section{Patients and Methods}

This prospective study included (30) patients in different age groups who were highly suspected with laryngeal tumors. Patients were referred by Oto Rhino Laryngology (ORL) Department of Tanta University Hospital to the CT Unit \& MRI Units of the Radiodiagnosis Department, Tanta University Hospitals, during the period from May 2016 to December 2017. Ethics Committee approval and informed consents were obtained. Privacy $\&$ confidentiality of all patient data were guaranteed. All data provision were monitored and used for scientific purpose only.

\section{The selection criteria were:}

Any patient who was suspected clinically by laryngeal complaint (e.g. hoarseness of voice, difficulty of swallowing, neck swelling) and/or known to be having a laryngeal tumor.

\section{Exclusion criteria:}

- Medical exclusion criteria:

- Impaired renal excretory function (calculated GFR less than $30 \mathrm{ml} /$ minute $/ 1.73 \mathrm{~m}^{2}$ ).

- History of allergy to contrast media.

- Patient who refuses the examination.

- CT exclusion criteria: Pregnancy.

- MRI exclusion criteria:

- Patients with intraocular metalic foreign body.

- Patients with MR non compatible intracranial clips of arterial brain aneurysms, cochlear implants or cardiac pacemakers.

- Claustrophobia.

\section{All patients were subjected to the following:}

1- Informed consent obtained from all patients.

2- Proper history taking.

3- Clinical examination: This was done at the ORL Department and included.

4- Laboratory investigations: Full blood screen and biochemical profile.

5- Radiological and medical imaging examinations: All the patients were subjected to CT examina- tion of the larynx while 22 patients were subjected to MR examination within maximum interval of 6 days.

\section{Technique of CT examination of the larynx:}

Evaluation of laryngeal tumors requires a contrast enhanced CT study of the neck. Images of the neck were obtained, examination was done for all patients in CT Unit, Diagnostic Radiology Department using multi detector CT (GE optima 660128 slice) followed by injection of an iodinated contrast agent (total dose $35-40 \mathrm{~g}, 1 \mathrm{ml} / \mathrm{kg}$ body weight). The contrast was injected by an automated power injector.

\section{Patient preparation and positioning:}

The patient lied in supine position with neck hyperextended, breathing quietly and is asked to refrain from coughing or swallowing.

Following acquisition of the lateral scout view, axial slices were obtained from the skull base to the aortic arch with the acquisition plane parallel to the plane of hyoid bone, to obtain scans parallel to the true vocal cords. The raw axial image dataset is reconstructed with a section thickness of about $0.75 \mathrm{~mm}$ to obtain high quality sagittal and coronal reformatted images. A 512 X 512 matrix is used with a small field of view (FOV) between $16 \mathrm{X}$ $16 \mathrm{~cm}$ was appropriate to cover the lymph nodes. All images were reviewed in soft tissue and bone windows.

\section{Technique of MR imaging of the larynx:}

MR imaging was done for 22 patients in MRI Unit, Diagnostic Radiology Department, Tanta University Hospitals. MRI scans were performed using high field MRI scanners (GE Signa Explorer 1.5-T closed magnet) using anterior neck coil. A combination of multi-planar non contrast T1weighted, T2-weighted and T2-weighted fat saturation images with post contrast $\mathrm{T} 1$ fat-suppressed images were routinely used. It is important to take the T1 and T2 sections at the same levels. A section thickness of $4 \mathrm{~mm}$ is preferred with an interslice gap of $0-1 \mathrm{~mm}$.

\section{Patient preparation and positioning:}

All patients were examined supine with the use of neck surface coil and immobilized in comfortable position.

\section{Pulse sequence and scanning plane:}

A scout T1 weighted sagittal image view to verify precise position of patient and act as a localizer of subsequent slices. 
Magnetic resonance imaging protocol included: Pre-contrast imaging:

A- An axial thin section T1W SE and T2W FSE images are obtained covering the larynx from the base of the tongue to the first tracheal ring, the scan orientation was parallel to the laryngeal ventricle.

- Axial T 1 WI (TR/TE=400-600/10-20m/sec).

- Axial T2WI (TR/TE=2000-4000/100-120 $\mathrm{m} / \mathrm{sec}$ ).

- Typical imaging parameters for standard examination:

- Section thickness of $4 \mathrm{~mm}$.

- Interslice gap of 0.5-1 mm.

- A field of view (20 X $20 \mathrm{~cm})$.

- An acquisition matrix of 256 X 192.

B- Coronal and/or axial FLAIR images (TR/TE/ Inversion time $(\mathrm{TI})=4000-6000 / 140 / 1200)$.

C- Sagittal T 1 W SE images were performed with sagittal sections in the true midline localized from axial pre-contrast scan.

\section{Post-contrast imaging:}

Post-contrast axial, coronal and sagittal fat saturated T1 WI study was done after injection of gadolinium-based contrast agents as Magnevist $(0.1 \mathrm{ml} / \mathrm{Kg})$.

6- Endoscopic examination was done for all patients and was carried under general anesthesia for direct examination, biopsy and histopathological examination.

7- Pre therapeutic staging: The tumor was staged according to TNM classification based on the combined clinical, radiological and histopathological data.

\section{Results}

This study included 30 patients who were highly suspected to have laryngeal tumors; 26 males and 4 females with age ranges from 40 years to 75 years (mean age 60.6 years). The affection with laryngeal tumors was predominant in males representing $86.7 \%$ of the total number of cases, shown in (Table 1).

The 30 patients in our study presented clinically by variety of symptoms, 18 patients presented mainly by hoarseness of voice, 10 patients with difficult painful swallowing or dysphagia while only 2 patients presented by neck swelling. In our study, hoarseness of voice was the main presenting symptom in 18 patients $(60 \%)$, followed by dys- phagia in 10 patients $(33.3 \%)$ while only 2 patients $(6.7 \%)$ presented by neck swelling.

Table (1): Age and gender distribution of the studied patients.

\begin{tabular}{|c|c|c|}
\hline \multirow{2}{*}{ Characteristics } & \multicolumn{2}{|c|}{$(\mathrm{No}=30)$} \\
\hline & $\mathrm{N}$. & $(\%)$ \\
\hline $\begin{array}{c}\text { Age (in years): } \\
40-<50 \\
50-<60 \\
60-<70 \\
70-<80\end{array}$ & $\begin{array}{l}2 \\
8 \\
14 \\
6\end{array}$ & $\begin{array}{l}6.7 \\
26.7 \\
46.7 \\
20.0\end{array}$ \\
\hline $\begin{array}{l}\text { Mean } \pm \text { S.D } \\
\text { Range }\end{array}$ & & \\
\hline $\begin{array}{l}\text { Gender: } \\
\text { Male } \\
\text { Female }\end{array}$ & $\begin{array}{l}26 \\
4\end{array}$ & $\begin{array}{l}86.7 \\
13.3\end{array}$ \\
\hline
\end{tabular}

Out of 30 patients in our study, 22 patients had performed combined CT and MRI followed by laryngoscopic examination, the remained 8 patients had performed CT only. Histopathological examination was done for all 30 cases.

In our study; 28 cases were histopathologically confirmed to be neoplastic (28/30) representing $93.3 \%$ of cases while only 2 cases showed inflammatory conditions (2/30) $6.7 \%$ of cases as shown in (Table 2).

Table (2): Aetiological classification according to histopathology of the 30 patients in our study.

\begin{tabular}{lll}
\hline Aetiology & $\mathrm{N}$. & $(\%)$ \\
\hline $\begin{array}{l}\text { Neoplastic } \\
\text { Non-neoplastic: } \\
\quad \text { Inflammatory } \\
\quad \text { Non inflammatory }\end{array}$ & 28 & 93.3 \\
\hline Total & 2 & 6.7 \\
\hline
\end{tabular}

Out of the 28 patients who had been proved histopathologically to be neoplastic lesions; 26 patients showed primary laryngeal neoplasms $(26 / 28)$ while only 2 patients showed metastatic deposits $(2 / 28)$.

Primary laryngeal tumors were the most common type encountered in our study (26/28 patients) representing $92.85 \%$ of cases while metastatic deposits represented only $7.15 \%$ ( $2 / 28$ cases): 1 from NHL and 1 from metastatic HCC.

The 28 patients diagnosed with laryngeal neoplasms were further classified according to the regional area of involvement into: 8 patients had transglottic carcinoma, 8 patients had glottic with supraglottic carcinoma, 6 patients had glottic carcinoma only and 6 patients with supraglottic carcinoma only. 


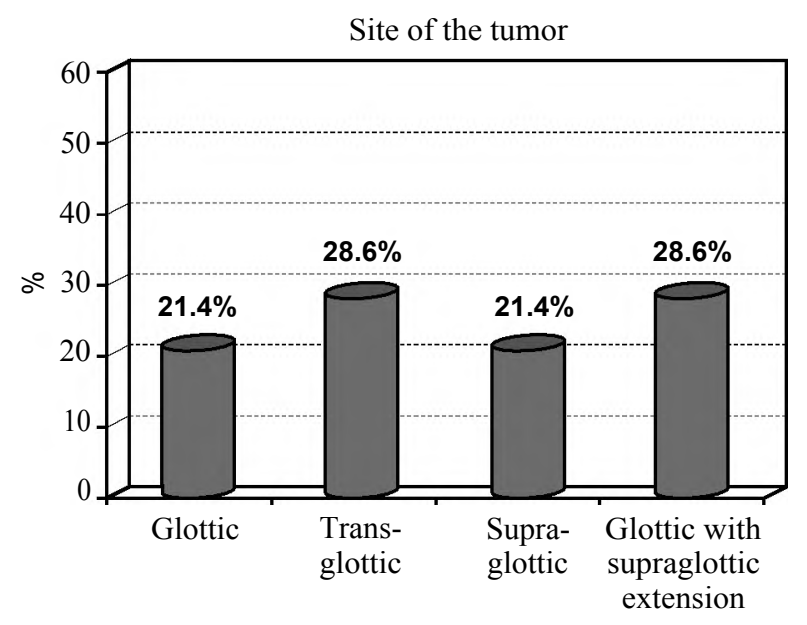

Fig. (1): The site of the tumor among the studied patients.

Transglottic and glottic with supraglottic extension of the tumors were the most common regions affected in our study, each represented $28.6 \%$ of cases. Glottic involvement noted in $78.6 \%$ of cases, No cases showed subglottic carcinoma only.

The histological findings showed 20 cases with squamous cell carcinoma, 2 cases with carcinoma in situ, 2 cases with adenocarcinoma, 2 with chondrosarcoma and 2 with metastatic deposits as shown in (Table 3).

Table (3): Histological classification of the 28 patients with laryngeal neoplasms.

\begin{tabular}{llll}
\hline Origin of the tumor & $\begin{array}{c}\text { Histopathological } \\
\text { diagnosis }\end{array}$ & N. & $(\%)$ \\
\hline Primary laryngeal & Squamous cell carcinoma & 20 & 71.6 \\
tumor (total 26) & Adenocarcinoma & 2 & 7.1 \\
& Chondrosarcoma & 2 & 7.1 \\
Metastatic laryngeal & NHL & 2 & 7.1 \\
tumor (total 2) & Metastatic HCC & 1 & 7.1 \\
\hline Total & & 1 & \\
\hline
\end{tabular}

Histopathological staging was done for 26 patients diagnosed with primary laryngeal neoplasms according to TN staging system. M staging wasn't included in our study.

Histopathological T staging of 26 cases with primary malignant laryngeal neoplasms; 2 cases showed carcinoma in situ, 4 cases with T1, 8 cases with T2, 4 cases with T3 and 8 cases with T4.

Histopathological $\mathrm{N}$ staging that was done for 15 cases ( 12 cases by block neck dissection while 3 cases by selective neck dissection, the other 15 cases showed non necessary neck dissection or nodal biopsy); 11 cases were N0 (no nodal involvement) and 4 cases with N1 (metastatic LNs sized less than $3 \mathrm{~cm}$ ).
In our study, the most frequent pathological $\mathrm{T}$ stage was T2 \& T4 representing $30.8 \%$ for each while the most frequent $\mathrm{N}$ stage was $\mathrm{N} 0$ representing $73.3 \%$.

CT examination of the neck was done for all 30 patients. CT findings were compared to histopathological staging of those patients as shown in (Table 4).

Table (4): Tumor T-staging by CT compared to histopathology.

\begin{tabular}{lccccccc}
\hline \multirow{2}{*}{ Histopathology } & \multicolumn{7}{c}{ CT } \\
\cline { 2 - 8 } & T0 & Tis & T1 & T2 & T3 & T4 & Total \\
\hline T0 & 2 & 0 & 0 & 0 & 0 & 0 & 2 \\
Tis & 2 & 0 & 0 & 0 & 0 & 0 & 2 \\
T1 & 0 & 0 & 4 & 0 & 0 & 0 & 4 \\
T2 & 0 & 0 & 0 & 8 & 0 & 0 & 8 \\
T3 & 0 & 0 & 2 & 0 & 0 & 2 & 4 \\
T4 & 0 & 0 & 0 & 2 & 0 & 6 & 8 \\
Secondary tumor & 0 & 0 & 2 & 0 & 0 & 0 & 2 \\
\hline Total & 6 & 0 & 6 & 10 & 0 & 8 & 30 \\
\hline
\end{tabular}

CT accurately staged 20 patients from the 30 patients underwent both CT \& histopathological T-staging (20/30). The total accuracy was $66.7 \%$. CT misdiagnosed 2 cases, over-staged 2 cases and under-staged 6 cases. CT couldn't detect the 2 cases with carcinoma in situ and was reported as normal study.

CT nodal staging was done for 15 cases; preoperative findings were correlated with postoperative histopathological data to obtain $\mathrm{N}$ staging accuracy of CT in those patients as shown in (Table $5)$.

Table (5): Tumor nodal (N-staging) by CT compared to histopathology.

\begin{tabular}{lccc}
\hline \multirow{2}{*}{ Histopathology } & \multicolumn{3}{c}{$\mathrm{CT}$} \\
\cline { 2 - 4 } & $\mathrm{N} 0$ & $\mathrm{~N} 1$ & Total \\
\hline N0 & 10 & 1 & 11 \\
N1 & 1 & 3 & 4 \\
\hline Total & 11 & 4 & 15 \\
\hline
\end{tabular}

CT accurately staged 13 patients from the 15 patients who underwent both CT \& histopathological N-staging (13/15) giving accuracy of $86.7 \%$. CT overstaged 1 case and understaged 1 case.

For assessment of the tumor extension by CT, CT findings were collected and classified according to the parts involved and the results were compared to histopathological findings as shown in (Table $6)$. 
Table (6): CT findings and regional tumoral infiltration in comparison to histopathological findings in 28 patients with laryngeal neoplasms.

\begin{tabular}{llcc}
\hline & \multicolumn{3}{c}{ No. of cases } \\
\cline { 2 - 4 } Regional involvement & By & By & Accuracy \\
& CT & pathology & 75 \\
\hline Pre-epiglottic space & 3 & 4 & 80 \\
Paraglottic space & 4 & 5 & 83.3 \\
Anterior commissure & 5 & 6 & 66.6 \\
Posterior commissure & 2 & 3 & 85.7 \\
Pyriform sinus & 6 & 7 & 80 \\
Epiglottis & 4 & 5 & 87.5 \\
True vocal cords & 14 & 16 & 71.4 \\
Thyroid cartilage & 5 & 7 & 85 \\
Subglottic region & 6 & 7 & 83.3 \\
Extra-laryngeal & 5 & 6 & \multicolumn{3}{c}{. } \\
\hline
\end{tabular}

CT showed accuracy $71.4 \%$ in detection of the thyroid cartilage invasion, $75 \%$ in detection of preepiglottic space infiltration, $80 \%$ of paraglottic space infiltration, $83.3 \%$ in detection of infiltration of anterior commissure, $85.7 \%$ in extension to pyriform sinus, $85 \%$ in subglottic extension, $83.3 \%$ in extra-laryngeal spread and $86.7 \%$ in metastatic lymph nodes.

Statistical analysis of the collected data was done to obtain CT sensitivity and specificity.

MRI diagnosed 26 cases from the 30 cases while misdiagnosed 2 cases giving sensitivity of $92.9 \%$ while properly diagnosed the 2 true negative cases on histopathology giving specificity of $100 \%$.

Table (7): Agreement Sensitivity and specificity for CT compared with histopathology in detection of laryngeal tumors.

\begin{tabular}{cccccccc}
\hline & \multicolumn{2}{l}{ Histopathological diagnosis } & Sesitivity \% & Specificity \% & PPV \% NPV \% \\
\cline { 2 - 4 } & Positive & Negative & Total & & & \\
\hline$C T:$ & 26 & 0 & 26 & $92.9 \%$ & $100.0 \%$ & $100.0 \%$ & $50.0 \%$ \\
Positive & 26 & 4 & & & & \\
Negative & 2 & 2 & 30 & & & \\
\hline Total & 28 & 2 & & & & \\
\hline
\end{tabular}

PPV: Positive Predictive Value.

NPV: Negative Predictive Value.

Pre and post-contrast MRI of the neck were done for 22 patients. MR findings were compared to histopathological staging of those patients.

Table (8): Tumor T-staging by MRI compared to histopathology.

\begin{tabular}{lcccccc}
\hline \multirow{2}{*}{ Histopathology } & \multicolumn{6}{c}{ MRI } \\
\cline { 2 - 7 } & Tis & T1 & T2 & T3 & T4 & Total \\
\hline Tis & 2 & 0 & 0 & 0 & 0 & 2 \\
T2 & 0 & 1 & 7 & 0 & 0 & 8 \\
T3 & 0 & 0 & 0 & 3 & 1 & 4 \\
T4 & 0 & 0 & 0 & 1 & 7 & 8 \\
\hline Total & 2 & 1 & 7 & 4 & 8 & 22 \\
\hline
\end{tabular}

MRI accurately staged 19 patients from 22 patients while understaged 2 cases and overstaged 1 case from who underwent MRI-T staging (19/22) giving accuracy of $86.7 \%$.

Table (9): Tumor N-staging by MRI compared to histopathology.

\begin{tabular}{lccc}
\hline \multirow{2}{*}{ Histopathology } & \multicolumn{3}{c}{ MRI } \\
\cline { 2 - 4 } & $\mathrm{N} 0$ & $\mathrm{~N} 1$ & Total \\
\hline N0 & 11 & 0 & 11 \\
N1 & 0 & 4 & 4 \\
\hline Total & 11 & 4 & 15 \\
\hline
\end{tabular}

Pre-operative MRI findings were correlated with post-operative histopathological data to obtain $\mathrm{N}$ staging accuracy of MRI in 15 patients.

MRI accurately staged 15 patients from 15 patients who underwent MRI-N staging (15/15) giving accuracy of $100 \%$.

For assessment of the tumor extension by MR, MRI findings were collected and classified according to the parts involved and the results were compared to post-operative pathological findings.

Table (10): MR findings and regional tumoral infiltration in comparison to histopathological findings in 22 patients with laryngeal neoplasms.

\begin{tabular}{lccc}
\hline & \multicolumn{3}{c}{ No. of cases } \\
\cline { 2 - 4 } Regional involvement & $\begin{array}{c}\text { By } \\
\text { MR }\end{array}$ & $\begin{array}{c}\text { By } \\
\text { pathology }\end{array}$ & $\begin{array}{c}\text { Accuracy } \\
\%\end{array}$ \\
\hline Pre-epiglottic space & 4 & 4 & $100 \%$ \\
Paraglottic space & 4 & 4 & $100 \%$ \\
Anterior commissure & 5 & 6 & 83.3 \\
Posterior commissure & 3 & 3 & 100 \\
Pyriform sinus & 6 & 7 & 85.7 \\
Epiglottis & 4 & 4 & 100 \\
True vocal cords & 14 & 14 & 100 \\
Thyroid cartilage & 6 & 7 & 85.7 \\
Subglottic region & 6 & 6 & 100 \\
Extralaryngeal & 5 & 5 & 100 \\
\hline
\end{tabular}


MR showed accuracy $85.7 \%$ in detection of the thyroid cartilage invasion, $100 \% \%$ in detection of pre-epiglottic space infiltration, $100 \%$ of paraglottic space infiltration, $83.3 \%$ in detection of infiltration of anterior commissure, $85.7 \%$ in extension to pyriform sinus, $100 \%$ in subglottic extension, $100 \%$ in extralaryngeal spread and $100 \%$ in metastatic lymph nodes.

Statistical analysis of the collected data was done to obtain MR sensitivity and specificity.

MRI diagnosed 21 cases from the 22 cases while misdiagnosed 1 case giving sensitivity of $95.5 \%$, while specificity of MR couldn't be calculated on statistical basis due to absence of negative cases proved by histopathology among 22 cases who were subjected to MR.
The overall CT and MR findings were collected and classified according to the regional infiltration and compared with the histopathological findings in all patients of our study:

For proper evaluation of accuracy of $\mathrm{CT}$ and MRI in regional tumoral infiltrations, findings were collected and classified then were compared to pathological findings in 22 patients who underwent both imaging modalities as shown in (Table 12).

Both CT and MRI were equal in evaluation of certain areas as anterior commissure and pyriform sinus while MRI showed higher accuracy in evaluation of other laryngeal subsites namely preepiglottic, paraglottic spaces, posterior commissure, epiglottis, vocal cords, thyroid cartilage extralaryngeal spread and metastatic lymphadenopathy.

Table (11): Agreement sensitivity, specificity and accuracy for MRI compared with histopathology in detection of laryngeal tumors.

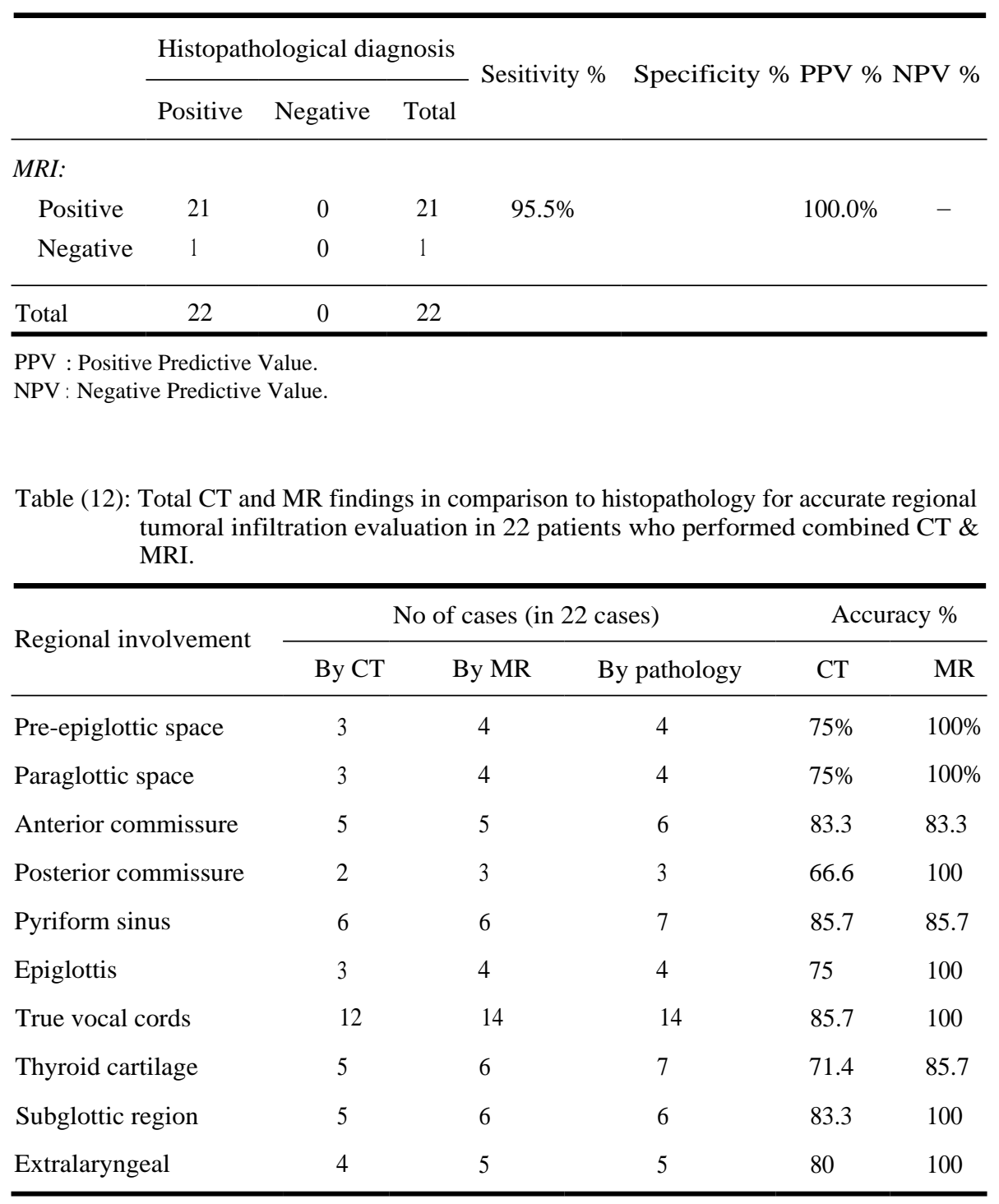




\section{Illustrated cases:}

\section{Case (1): Glottic carcinoma in situ:}
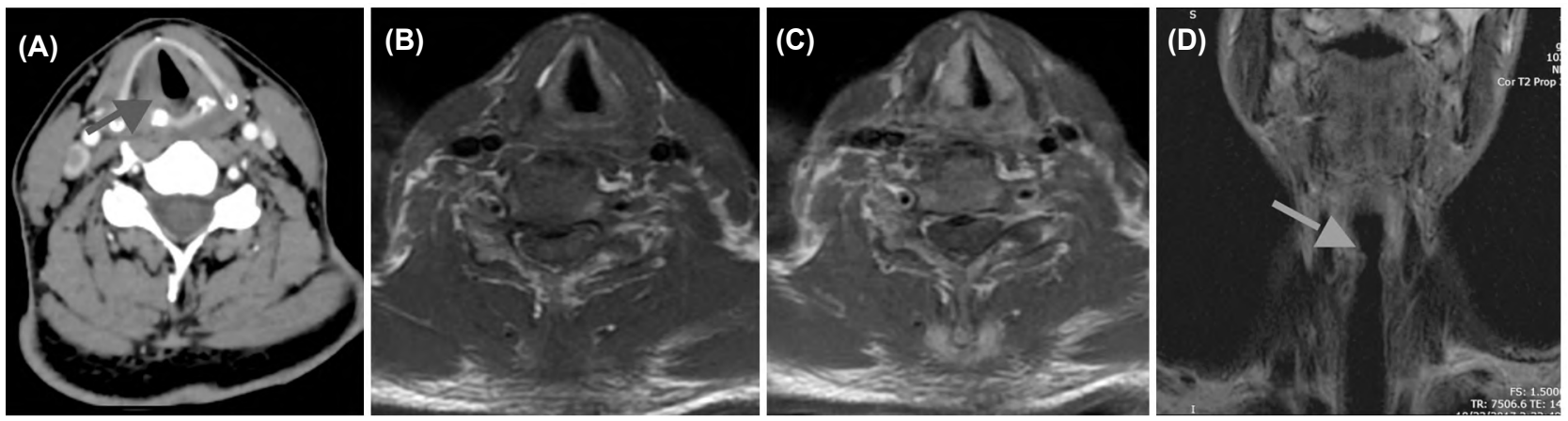

Fig. (2): (A,B,C and D), Male patient aged 70 years smoker, presented by hoaresness of voice. (A) Post-contrast CT Axial view at the glottic level, (B) Axial pre-contrast T1WI (C) Axial post-contrast T1WI (D) Coronal T2 WI FSE. Post-contrast CT showed no significant abnormality and was reported as normal study. MRI revealed minimal thickening of the right vocal cord is noted (B and D) with relative homogenous post-contrast enhancement of both vocal cords (C). Endoscopic biopsy and histopathology revealed carcinoma in situ.

Case (2): Glottic SCC:
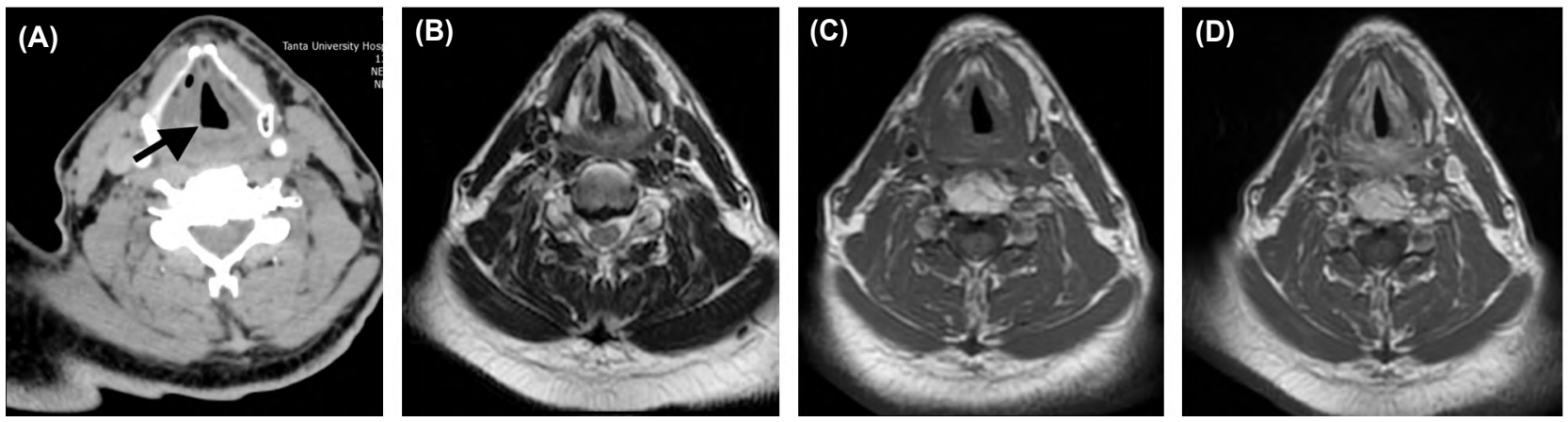

Fig. (3): (A, B, C and D); A Male patient aged 55 years presented with hoarsness of voice. (A) Post-contrast CT axial view at the glottic region, (B) Axial T1-weighted image. (C) Axial T2-weighted image. (D) Axial post-contrast T1-weighted image. CT neck with contrast revealed asymetrical right vocal cord thickening (arrow), no invasion of thyroid cartilage, anterior or posterior commissures. (A) On MRI mild thickening of the right vocal cord is noted (arrow) with preserved fat signal of the ipsilateral paraglottic space (B) A note is done for the homogenous signal of the vocal cord at normal side when compared to heterogeneous signal on the affected side (C) with relative enhancement of both vocal cords that seen slightly heterogeneous on the right side (D).

\section{Case (3): Metastasis of the thyroid cartilage:}
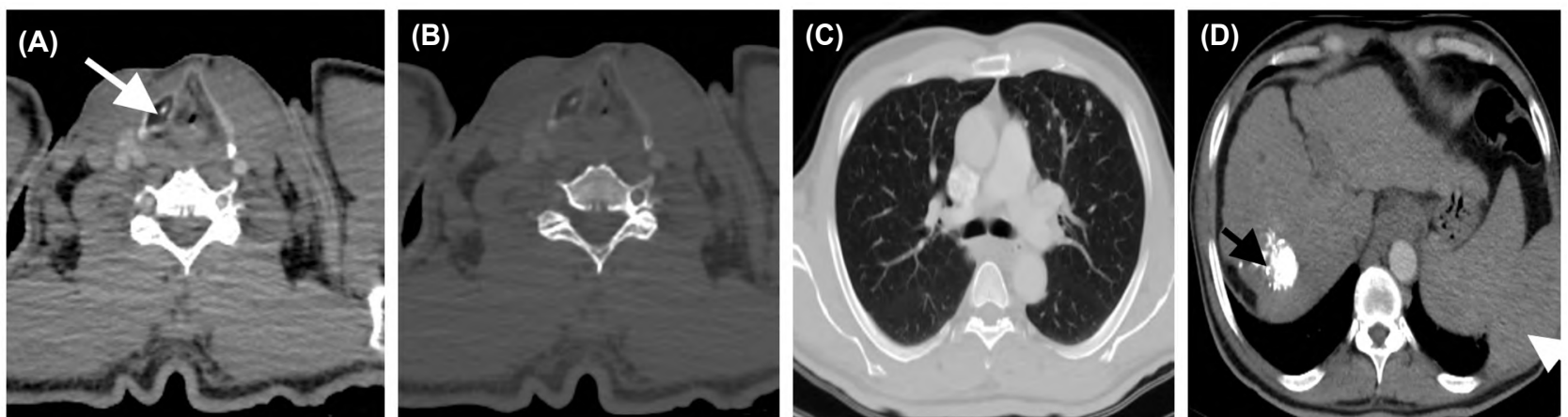

Fig. (4): Post-contrast CT of the Neck, Chest \& Abdomen) (A, B, C and D) A Male patient aged 70 years, known case of metastatic hepatocellular carcinoma underwent Trans-Arterial Chemoembolization (TACE). (A) Axial views, soft window at glottic level, (B) Bone window at the same level. (C) Axial CT Chest cuts (lung window) (D) Upper abdomen cuts revealed an expansile hypodense lesion noted at the right lamina of the thyroid cartilage (A,B), the chemoembolized hepatic focal lesion (black arrow) is seen at upper abdomen cuts (D), with a metastatic rib lesion at the left $8^{\text {th }}$ rib (white arrow), multiple pulmonary nodules scattered at both lungs (C) (arrow). 


\section{Discussion}

Laryngeal cancer is the most common cancer of the upper aerodigestive tract and the second most common cancer of the respiratory tract after lung cancer. Squamous Cell Carcinoma (SCC) is the most common cancer of the larynx and accounts for $90 \%$ of malignant laryngeal tumors. Supraglottic SCC after glottic SCC is the second most common malignant tumor of the larynx [10]

In the present study, it was found that the majority of patients were in fifth and sixth decades of life with mean age of 60.9 years. This agreed with Jian et al., [11] in their study with mean age of 61.5 years.

This study was done on 30 patients, 26 males $(86.7 \%)$ and 4 females $(13.3 \%)$ with male to female ratio 6.5:1, this agreed with Brady et al., [12] who reported male predominance in different laryngeal neoplasms with male to female ratio 6.9:1.

As regard clinical presentation, in this study 18 patients $(60 \%)$ complained mainly of hoarseness of voice, 10 patients (33.3\%) complained of dysphagia and 2 patients $(6.7 \%)$ presented with neck swelling. This agreed with Allegra et al., [13] who reported hoarseness of voice as the most common symptoms in different laryngeal neoplasms.

In this present study, $93.3 \%$ of the laryngeal lesions were neoplasms (28/30) where as $6.7 \%$ (2/30) were non-neoplastic lesions. Primary neoplasms were the most common neoplasms representing $92.85 \%$ of total number of cases (26/28).

As regards to the site of the tumors at different laryngeal compartments: $28.6 \%$ of patients had transglottic extension: $28.6 \%$ had glottic with supra glottic extension, $21.4 \%$ had glottic only and $21.4 \%$ had supra glottic only. Glottic involvement is noted in $78.6 \%$ of cases. However, Hazem et al., [14] in their study that was carried out on 295 patients with laryngeal SCC reported that and the most common tumors were glottic-supraglottic (62.4\%), transglottic (20.7\%), glottic only (10.8\%), supraglottic only (5.1\%), and finally subglottic (1.0\%), which is a rare subsite for laryngeal cancer.

As regards to the histological diagnosis: $71.6 \%$ of the primary laryngeal tumors were of squamous cell type (SCCs), 7.1\% were chondrosarcomas while $7.1 \%$ were adenocarcinomas and $7.1 \%$ were CIS, this agreed with Warner et al., [15] who reported in their study the histologic predominance of squamous cell carcinomas in all head and neck tumors.
As regards to pathological (T) staging of the primary laryngeal tumors: $30.8 \%$ were found to be T2, 30.8\% were $\mathrm{T} 4,15.3 \%$ were stage $\mathrm{T} 1,15.3 \%$ were stage T3 and 7.8 were Tis (carcinoma in situ), Jian et al., [11] also found in their study that pathological $\mathrm{T} 2$ constituted $38.4 \%$ followed by T3 while T4 stage represented $15.7 \%$.

As regards to $\mathrm{N}$ (nodal) staging $73.3 \%$ were stage N0 (no nodal involvement) while $26.7 \%$ were stage N1 (nodal size less than $3 \mathrm{~cm}$ ), this matched with Maziar et al., [10] who found that the rate of cervical nodal metastasis in laryngeal cancer specially supraglottic type varied from $16.7 \%$ to $54.5 \%$ in T1 and T4 patients, respectively, but there was no statistically significant association between tumor grade and cervical lymph node metastasis.

Computed Tomography (CT) used to be the examination of choice that is performed for the evaluation of pathological laryngeal lesions due to its wide accessibility, shorter scanning time as nearly free of movement artifacts, aimed especially for patients who require immediate diagnosis (large obstructive lesions) or for uncooperative patients [6].

Computed tomography is also a good tool for the evaluation of the cartilaginous skeleton of the larynx and the extra-laryngeal soft tissues as well but its diagnostic accuracy is lower than MR, which is important for surgery planning and for the assessment of the prognosis of radiotherapy Precise differentiation of soft tissues, especially in the area of pharynx and larynx, is limited due to lower soft tissue contrast and also influenced by many artifacts as dental restorations [16]

According to pathological $\mathrm{T}$ staging, the $\mathrm{T}$ staging accuracies of CT and MRI were $66.7 \%$ and $86.4 \%$ respectively, this matched with Jian et al., [11] who reported in their study that $\mathrm{T}$ staging accuracies of CT and MRI were $57.69 \%$ and $88.46 \%$ respectively, where a significant difference between both modalities was revealed by $(\mathrm{McNe}-$ mar's test) $(p<0.01)$.

Whereas the nodal $(\mathrm{N})$ staging accuracies of $\mathrm{CT}$ and MRI in our study were $86.7 \%$ (13/15) and $100 \%(15 / 15)$ respectively. However, Rubia et al., [17] reported that diagnostic accuracy of MDCT in nodal staging of head and neck tumor was $95.3 \%$ in their study.

Magnetic Resonance Imaging (MRI) characterized by significantly higher soft tissue resolution and possibility of multi-planar imaging in comparison to $\mathrm{CT}$, which enables better assessment of the 
range of pathological lesions, and the evaluation of submucosal spread of pathological processes. In MRI the patient is not exposed to ionizing radiation, and therefore, can be used for monitoring disease development and treatment efficacy $[7,16]$

The main limitations of MRI were the relatively long scanning time, which increases the possibility of the occurrence of movement artifacts, especially in patients with advanced lesions or obstruction of the airways. Others include artifacts associated with pulsatile movements of vessels, swallowing, and, above all, patient's breathing during scanning. It also required the cooperation and education of the patient-shallow breathing during scanning, as well as with holding cough and swallowing [16] .

Our study revealed that MRI was more accurate than CT scan in T staging of laryngeal tumors, MRI showed a higher sensitivity (95.5\%) and a higher accuracy $(86.4 \%)$ compared to $(92.9 \%)$ and (66.7\%) of CT, however, the results of MRI especially DWI scan was more likely to be influenced by examination time and movements such as breathing and swallowing. For patients with obstructive dyspnea or low compliance, large artifacts could be seen on MRI that was noted in 4 of our 22 patients who underwent MR examination (2 patients were uncooperative, 1 patient with irregular breathing due to large obstructive mass and 1 with tracheostomy tube inserted), causing incorrect interpretation of tumor extension.

Both Magnetic Resonance Imaging (MRI) and Computed Tomography (CT) can be used to assess cartilage invasion preoperatively. CT and MRI each have their unique strengths and weaknesses. MRI provides good contrast between different soft tissues, which makes it superior in distinguishing between soft tissue and tumour. However, cartilage invasion is easily overestimated, since it is hard to distinguish from peri-tumoral inflammation [18]

This prospective study evaluated the role CT and MRI in the evaluation of different laryngeal tumors specially cancers as the proper evaluation of mucosal and submucosal areas that would change the stage of the disease and the choice of therapeutic approach. In particular, MRI showed an accuracy of $100 \%$ in assessing areas such as paraglottic, pre epiglottic spaces as well as extra-laryngeal spread and metastatic lymph nodes. In our study, CT staging was accurate in $66.7 \%$ of cases, while the MRI was accurate in $86.7 \%$ of cases as in comparison to histopathological data.

The comparison of diagnostic accuracy of CT (in 28 cases) and MRI (in 22 cases) for different laryngeal subsites such as PES, PGS, ATC, TC were compared to results of Aniruddha et al., [19]

In Pre-Epiglottic Space (PES) involvement: Diagnostic accuracy of CT and MRI were $75 \%$ and $100 \%$ respectively while Aniruddha et al., [19] reported that diagnostic accuracy were relatively higher in CT than MRI (CT 94.7\% while $89.5 \%$ for MRI).

In Para-Glottic Space (PGS) involvement: Diagnostic accuracy of CT and MRI were $80 \%$ and $100 \%$ respectively while Aniruddha et al., [19] reported that diagnostic accuracy were relatively higher in MRI than CT (CT $89.5 \%$ while $94.7 \%$ for MRI).

In anterior commissure (ATC) involvement: Diagnostic accuracy of CT and MRI were the same 83.3\% for each while Aniruddha et al., [19] reported that diagnostic accuracy were slightly higher in MRI than CT (CT $84.2 \%$ while $89.5 \%$ in MRI).

In Thyroid Cartilage (TC) invasion: Diagnostic accuracy of CT and MRI were $71.4 \%$ and $85.7 \%$ respectively while Aniruddha et al., [19] reported that diagnostic accuracy of CT $78.9 \%$ while $84.2 \%$ for MRI.

\section{Conclusion:}

Each of the two imaging techniques has their own advantages and disadvantages on determining the local infiltration and extension of tumors. Combined utility of CT and MRI could help to overcome the disadvantages and improve the accuracy specially about thyroid cartilage assessment and $\mathrm{T}$ staging of laryngeal carcinomas.

MR imaging has several advantages with respect to multi-slice CT and few limitations (artifacts related to movement). MRI allows a multiparameter analysis (T1 weighted, T2 weighted, DWI, and post contrast acquisition). This multiparameter approach amplifies the contrast resolution, even though MRI is more expensive, longer, and not always feasible for patients compared to CT scan (poor compliance, any contraindications), based on the above considerations, we believe that MRI could be considered the investigation of choice in the clinical evaluation of laryngeal tumors specially early glottic lesions for the planning of therapeutic interventions, because of its high sensitivity and elevated degree of diagnostic accuracy.

\section{Recommendations:}

MRI should be considered the preliminary imaging modality in evaluation of different laryngeal tumors specially early glottic tumors. CT 
examination alone could be used as an alternative in few circumstances when MR is not feasible.

\section{References}

1- PARKIN M.D., BARY F., FERLAY J., et al.: Global cancer statistics. CA cancer J. Clin., 55: 74-108, 2005.

2- HASHIBE M., BOFETTA P., ZARIDZE D., et al.: Contribution of tobacco and alcohol to the high rates of squamous cell carcinoma of the supraglottis and glottis in Europe. Am. J. Epidemiology, 165: 814-20, 2007.

3- CONNOR S.: Laryngeal cancer: How does the radiologist help? Cancer Imaging, 28: 93-103, 2007.

4- BECKER M., LEUCHTER I., PLATON A., et al.: Imaging of laryngeal trauma. Eur. Journal of Radiology, 83: 14254, 2014.

5- KIM J.W., ROH J.L., KIM J.S., et al.: Evaluation of 18FFDG PET/CT and CT/MRI with histopathological correlation in patients undergoing compartment neck dissection for squamous cell carcinoma of the larynx, hypopharynx and esophagus. Oral. Onchol., 49: 449-53, 2013.

6- HERMANS R.: Diagnostic Imaging. In: Head and Neck Cancer Imaging. Hermans R. 2 nd ed. Springer; Berlin, 55-95, 2012.

7- HUANG B.Y., SOLLE M. and WEISSLER M.C.: Larynx: Anatomic imaging for diagnosis and management. Otolaryngol. Clinc. North Am., 45: 1325-61, 2012.

8- BECKER M., BURKHARDT K., DULGUEROV P., et al.: Imaging of the larynx and hypopharynx. European Journal of Radiology, 66: 450-79, 2008.

9- MAROLDI R., RAVANELLI M. and FARINA D.: Current opinion in Otolaryngology, Head \& Neck Surgery, 22: 131-9, 2014.

10- MAZIAR M.L., BEHROOZ A., AMIN A., et al.: Rate of Occult Cervical Lymph Node Involvement in Supraglottic
Squamous Cell Carcinoma. Indian Journal of Otorhinolaryngology, 29: 133-6, 2017.

11- JIAN H.W., JING Z., ZENG H.L., et al.: Comparison of CT and MRI in Diagnosis of Laryngeal Carcinoma with Anterior Vocal Commissure Involvement. Scientific reports, 6: 1-7, 2016.

12- BRADY J.S., MARCHIANO E., KAM D., et al.: Survival Impact of Initial Therapy in Patients with T1-T2 Glottic Squamous Cell Carcinoma .Otolaryngology-Head and Neck Surgery, 155: 257-64, 2016.

13- ALLEGRA E., FERRISE P., TRAPASSO S., et al.: Early Glottic Cancer: Role of MRI in the Preoperative Staging. Bio Medical Research International, 10: 1155-62, 2014.

14- HAZEM M., MIKHAIL W., NASSER A., et al.: Study of the epidemiology and management of laryngeal cancer in Kasr Al-Aini Hospital .Egyptian Journal of Otorhinolaryngology, 30: 208-14, 2014.

15- WARNER L., CHUDASAMA J., KELLY C.G., et al.: Radiotherapy versus open surgery versus endolaryngeal surgery (with or without laser) for early laryngeal squamous cell cancer. Cochrane Database Syst. Rev., 12: 45163, 2014.

16- NINA B.K., WIOLETTA P., PIOTR G., et al.: Rare Infraglottic Lesions in Magnetic Resonance Imaging. Polish Journal of Radiology, 79: 305-10, 2014.

17- RUBIA S., MUNAWAR H. and SYED O.A.: Diagnostic Accuracy of Multislice CT in the detection of Occult Cervical Lymph Node Metastasis in Head and Neck Cancers: Nodal CT staging. Journal of the college of physicians and surgeons Pakistan, 27: 275-7, 2017.

18- MAROLDI R., RAVANELLI M. and FARINA D.: Magnetic resonance for laryngeal cancer. Current Opinion in Otolaryngology \& Head and Neck Surgery, 22: 131-9, 2014.

19- ANIRUDDHA S., NISHI S., ANIL R., et al.: Role of computed tomography, magnetic resonance imaging, and endoscopy in pretherapeutic evaluation of laryngeal tumors. ENT Oncology, 2: 172-8, 2016. 


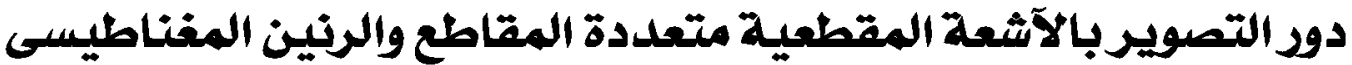

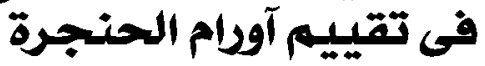

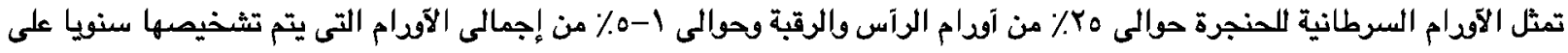

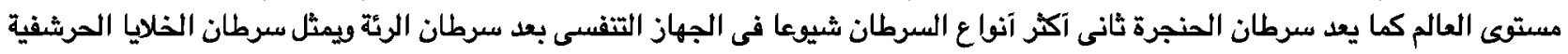

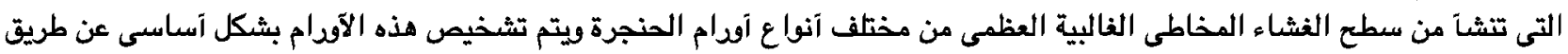

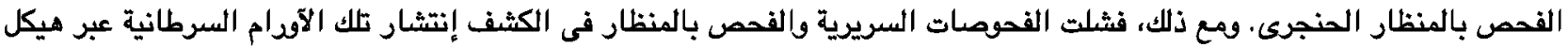
الحنجرة إلى خارجها مما يؤدى إلى التقليل المرحلى لتلك الآددام المتقدمة سريريا.

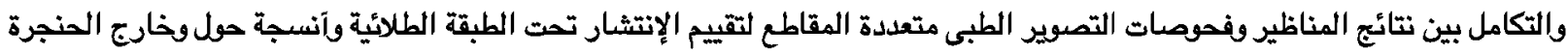

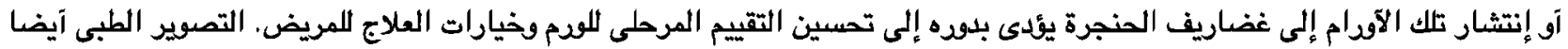

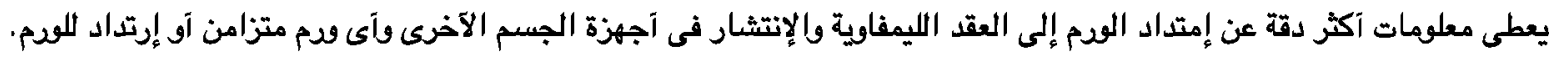

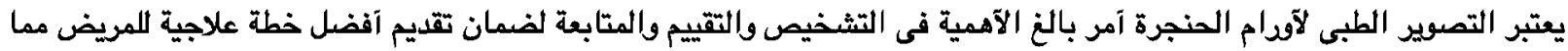
يؤدى إلى تقليل المضاعفات والوصول إلى آعلى معدلات الشفاء كما تساعد فى تحديد آنسب مو قع لآخذ العينة لتاكيد التشخيص.

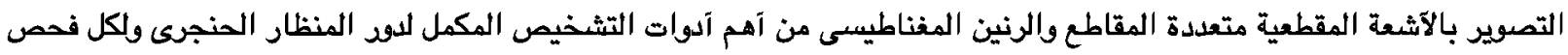

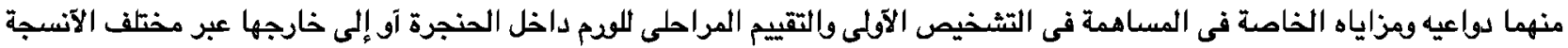

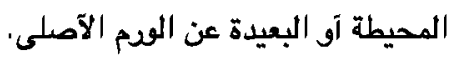

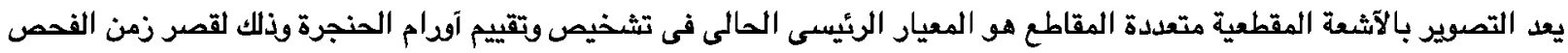

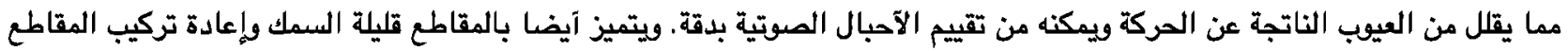

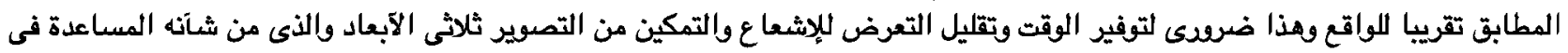

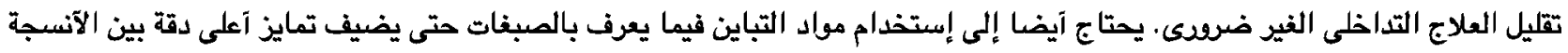

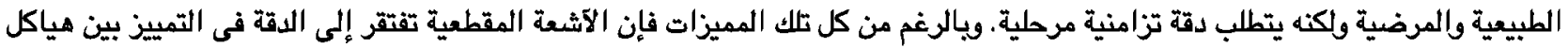

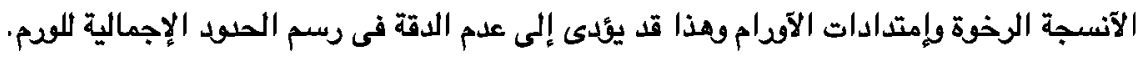

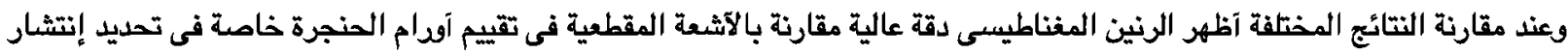

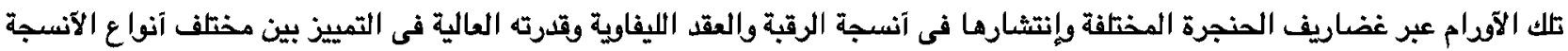

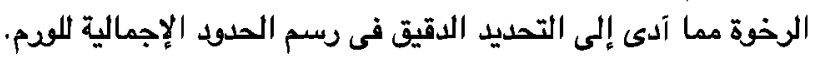

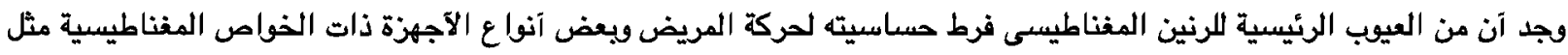

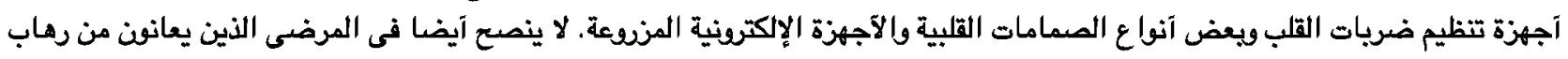

الآلاكاكن المغلقة.

ومن خلال تحليل نتائج هذه الدراسة إتضح الآتى:

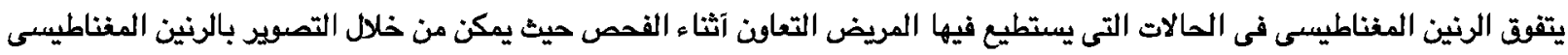

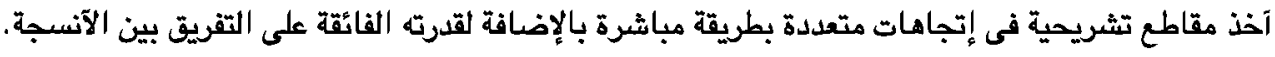
يسمح الفحص بالرنين المفناطيسى بوئية العضلات الداخلية الحنجرة والتى تعتبر مهمة فى تحديد الإمتداد السرطانى. بالإضافة إلى ذلك فإن التصوير عن طريق الرنين المفناطيسى آكثر دقة من الآشعة المقطعية فى تصديد مدى الإمتداد السرطانى إلى داخل 